\title{
Estabilidade química, físico-química e microbiológica de polpas de acerola pasteurizadas e não-pasteurizadas de cultivo orgânico
}

\author{
Chemical, physicochemical and microbiological stability of pasteurized and non pasteurized \\ acerola pulps from organic cultivation \\ Rafaela Maria Temóteo LimaI ${ }^{\mathrm{I}}$ Raimundo Wilane de Figueiredo ${ }^{\mathrm{II}}$ Geraldo Arraes Maia ${ }^{\mathrm{II}}$ \\ Paulo Henrique Machado de Sousa ${ }^{\text {II }}$ Evânia Altina Teixeira de Figueiredo ${ }^{\text {II }}$ \\ Cinthia Soares Rodrigues ${ }^{\text {II }}$
}

RESUMO

O presente trabalho objetivou avaliar a estabilidade química, físico-química e microbiológica de polpas de acerola pasteurizadas e não-pasteurizadas, oriundas de cultivo orgânico e armazenadas sob congelamento $\left(-18 \pm 2^{\circ} \mathrm{C}\right)$ durante 360 dias. $O$ armazenamento sob congelamento não ocasionou perdas significativas de qualidade das polpas de acerola. No entanto, o tratamento térmico influenciou negativamente nos conteúdos iniciais de alguns componentes, principalmente sólidos solúveis, açúcares solúveis totais e redutores, que apresentaram conteúdos inferiores no início do armazenamento para as polpas pasteurizadas. As polpas pasteurizadas e não-pasteurizadas apresentaram boa qualidade microbiológica do início ao final do armazenamento. Dentre as polpas estudadas, as polpas não-pasteurizadas apresentaram melhores características iniciais de cor. As polpas pasteurizadas garantiram melhores características microbiológicas no que concerne aos aspectos de segurança alimentar.

Palavras-chave: acerola, polpa, cultivo orgânico, estabilidade, pasteurização, Malpighia emarginata.

\section{ABSTRACT}

The present research aimed to evaluate the chemical, physical-chemical and microbiological stability of pasteurized and non-pasteurized acerola pulp coming from organic cultivation stored frozen for 360 days. Storage under freezing did not cause significant loss of acerola pulp's quality. However, the heat treatment had a negative influence on the initial contents of some components, especially soluble solids, total soluble and reducing sugars, which showed lower contents at the beginning of storage for pasteurized pulp. Among the pulps studied, non-pasteurized pulps showed the best characteristics of the initial color. The pasteurized pulps guaranteed best features in regard to the microbiological aspects of food safety.

Key words: acerola, pulp, organic cultivation, stability, pasteurization, Malpighia emarginata.

\section{INTRODUÇÃO}

A acerola (Malpighia emarginata D.C.) ganhou um grande interesse agrícola relativo ao alto teor de vitamina $\mathrm{C}$ e aparência, além de sua larga capacidade de utilização industrial (FREITAS et al., 2006). A acerola apresenta-se como alternativa comercial altamente viável no mercado fruticultor e, pela superprodução que tem sido gerada, há uma justificativa aos estudos direcionados ao desenvolvimento de novos produtos a partir dessa matéria-prima, que concentra na fruta in natura e na polpa sua maior forma de consumo (SOARES et al., 2001).

A atividade agrícola mundial vem sendo diretamente influenciada pela preocupação da população, que busca hoje em seus produtos qualidade e segurança alimentar asseguradas. Simultaneamente, a proteção e a conservação do meio ambiente com uso de tecnologias mais limpas, têm conscientizado produtores no manejo do solo e formas de cultivo. Com a crescente demanda interna e externa por frutas

Instituto Federal de Educação, Ciência e Tecnologia do Ceará (IFCE), Av. Ouvidor Mor Vitorino Soares Barbosa, s/n, Saranhão, 62930-000, Baturité, CE, Brasil. E-mail: rtemoteo@ifce.edu.br. Autor para correspondência.

IIPrograma de Pós-graduação em Ciência e Tecnologia de Alimentos, Universidade Federal do Ceará (UFC), Fortaleza, Ceará, Brasil. 
produzidas em sistemas orgânicos, busca-se não apenas produtos saudáveis e de elevado valor nutricional, isentos de qualquer tipo de contaminantes que ponham em risco a vida do consumidor e do agricultor e o meio ambiente, mas também a preservação e ampliação da biodiversidade dos ecossistemas, a conservação das condições físicas, químicas e biológicas do solo e da qualidade da água e do ar (BORGES et al., 2003).

Diante dos fatos expostos, este trabalho teve como objetivo avaliar a estabilidade química, físicoquímica e microbiológica de polpas de acerola oriundas de cultivo orgânico, submetidas ou não ao tratamento de pasteurização e mantidas por 360 dias sob congelamento.

\section{MATERIAL E MÉTODOS}

As polpas de acerola foram obtidas a partir de frutos de seis clones de aceroleiras (Malpighia emarginata D.C.), a saber: AC 71, AC 69, FP 19, OKINAWA, I 13/2 e II 26/4, provenientes da fazenda de cultivo orgânico de uma empresa localizada no município de Ubajara-CE. Os frutos foram cuidadosamente colhidos em estádio maduro, transportados imediatamente para a unidade industrial de processamento de frutas da mesma empresa para a obtenção das polpas. Estas foram obtidas de acordo com o fluxograma apresentado na figura 1. Os frutos foram recepcionados, selecionados e pesados, com posterior pré-lavagem e imersão em água potável com teores de cloro entre 0,5 e $1,0 \mathrm{mg} \mathrm{L}^{-1}$. Os frutos foram enxaguados, novamente selecionados e processados em moinhos. Após o processamento, as polpas sofreram um pré-resfriamento e, logo após, foram pasteurizadas em pasteurizador de placas a uma temperatura de $95 \pm 3^{\circ} \mathrm{C}$ por 11 segundos. Após o tratamento, as polpas foram imediatamente envasadas em potes de polipropileno de $250 \mathrm{~g}$, submetidas a um congelamento rápido e armazenadas a temperaturas entre $-15^{\circ} \mathrm{C}$ e $-18^{\circ} \mathrm{C}$. Para as polpas que não sofreram tratamento térmico, após o pré-resfriamento, passouse diretamente para a etapa de envase, seguindo os mesmos procedimentos realizados para as polpas pasteurizadas. As amostras foram analisadas um dia após a obtenção da polpa e sequencialmente a cada 45 dias para avaliações físico-químicas, químicas e microbiológicas, em um período de 12 meses de armazenamento.

\section{Análises físicas e físico-químicas}

As polpas foram analisadas imediatamente após o processamento (tempo zero) e em intervalos

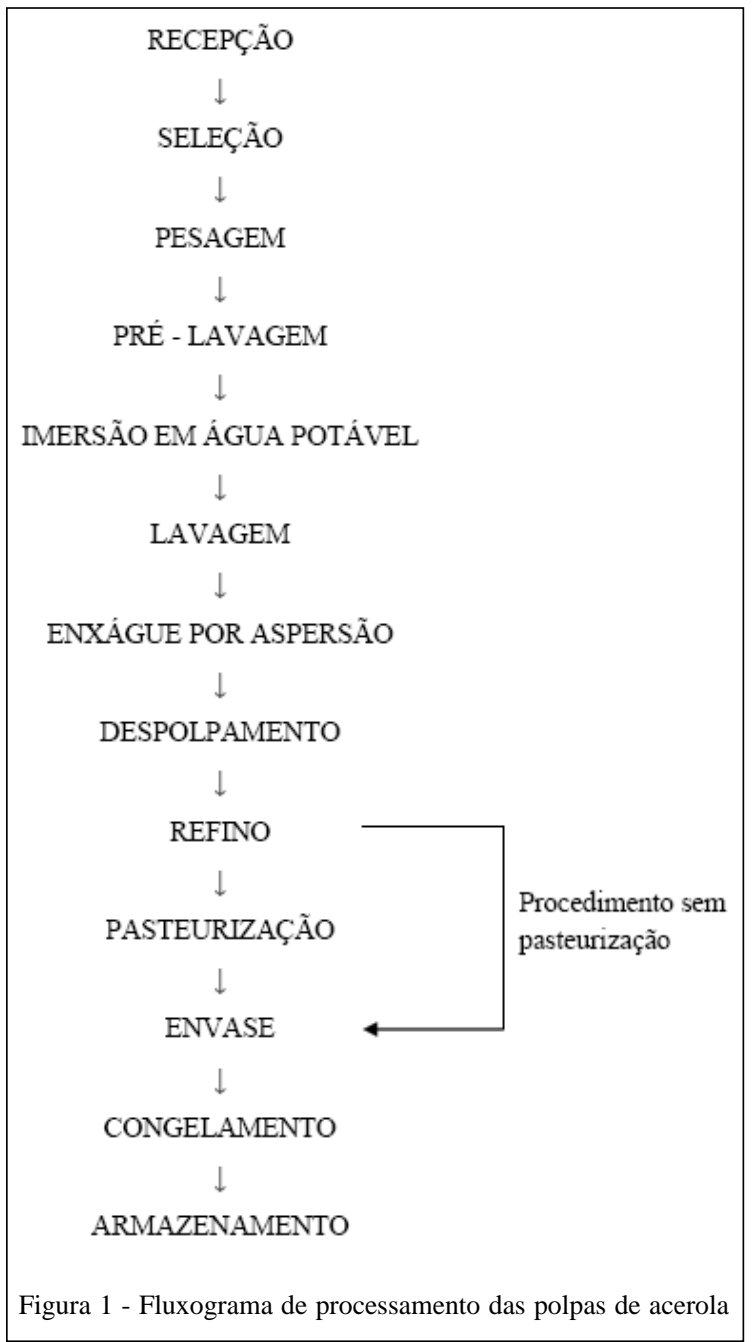

regulares de 45 dias para medir, em duplicata, os seguintes parâmetros: $\mathrm{pH}$ e sólidos solúveis totais (em refratômetro de mesa modelo Reichert AR 200), de acordo com AOAC (1995), acidez titulável em ácido cítrico e relação sólidos solúveis totais e acidez titulável, por BRASIL (2005), açúcares redutores e açúcares totais, conforme metodologia descrita por MILLER (1959), cor instrumental, utilizando colorímetro Konica Minolta Spectrophotometer CM-3500d.

\section{Análises microbiológicas}

As análises microbiológicas consistiram de contagem de coliformes totais, bactérias aeróbicas mesófilas, bolores e leveduras e determinação de presença de Salmonella sp. em todos os tempos de armazenamento, usando amostras em potes de polipropileno com $250 \mathrm{~g}$ e metodologias de acordo com as propostas por APHA (2001). 
Análise estatística

O experimento seguiu um delineamento em blocos casualizados em parcelas subdivididas, com dois tratamentos nas parcelas (polpas pasteurizadas e não-pasteurizadas) e nove tempos de armazenamento nas subparcelas $(0,45,90,135,180,225,270,315$ e 360 dias), mantidos a temperatura de congelamento $\left(-18^{\circ} \mathrm{C}\right)$ em fatorial inteiramente ao acaso, com três repetições do experimento. Os resultados químicos e físicoquímicos foram submetidos à análise de interação entre tratamentos e tempos de armazenamento e regressão e, quando conveniente, foi realizado teste de Tukey para comparação de médias, ao nível de $5 \%$ de probabilidade através do programa estatístico SAS versão 8.1 (2006).

\section{RESULTADOS E DISCUSSÃO}

Análises físicas e físico-químicas

A análise de variância das características químicas e físico-químicas detectou interações significativas $(\mathrm{P} \leq 0,05)$ entre os tratamentos e o tempo de armazenamento para luminosidade $\left(\mathrm{L}^{*}\right)$ e $\mathrm{pH}$. Portanto, para esses parâmetros, foi feita uma análise de regressão para cada tratamento separadamente. Para os parâmetros a*, b*, Chroma (c), ângulo Hue (h), sólidos solúveis (SS), acidez titulável (AT), relação sólidos solúveis e acidez titulável (SS/AT), açúcares solúveis totais e açúcares redutores, não foram detectadas interações significativas $(P>0,05)$ entre tratamento e tempo de armazenamento, sendo as avaliações do tempo de armazenamento realizadas conjuntamente para os dois tratamentos.

A cor é um atributo de qualidade para frutos destinados ao processamento, variando com a época de colheita, estádio de maturação e exposição solar. A determinação instrumental da cor é interessante para o estudo da variação e comparação com os pigmentos presentes nos frutos. A coloração vermelha forte é afetada pelo conteúdo total de antocianinas e sua distribuição, pela quantidade de cromoplastos que armazenam tais pigmentos pela formação de complexos antocianinas-metais e pelo pH (CHITARRA \& CHITARRA, 2005).

Os valores de $L^{*}$ permaneceram estáveis ao longo do tempo de armazenamento (Figura 2A). As polpas pasteurizadas apresentaram média de 45,46; enquanto as polpas não-pasteurizadas apresentaram média de 38,23. ARAÚJO (2005), ao estudar a estabilidade de polpas de frutos de clones de aceroleira oriundas de cultivo convencional por 12 meses sob congelamento, observou um aumento gradativo do valor L* em todos os clones durante o armazenamento.
O autor também relatou que as polpas dos clones que possuíam maiores quantidades de antocianinas, portanto mais escuras, apresentaram maior aumento da luminosidade, possivelmente devido à maior degradação deste pigmento. BRUNINI et al. (2004) analisaram acerolas provenientes de várias regiões de cultivo e observaram variações de 22,12 a 43,27.

As polpas de acerola apresentaram valores médios para a coordenada a*, que decresceram de 32,56, no início de armazenamento, para 16,09 ao final do armazenamento de 360 dias sob congelamento (Figura 2B). SILVA (2008) observou, para a coordenada a*, uma amplitude entre 10,54 e 46,13, com média de 30,44 em frutos de aceroleira oriundas de cultivo orgânico e convencional. Os valores da coordenada b* das polpas de acerola apresentaram uma pequena variação de 33,31 a 34,25 ao longo dos 360 dias de armazenamento sob congelamento, com média de 36,29. Nesta coordenada, a cor varia numa escala que vai do amarelo ao azul, estando os valores mais altos relacionados com o amarelo. Estes valores confirmam as observações realizadas nas polpas durante o armazenamento. As polpas pasteurizadas apresentaram coloração amarelada, com tendência ao amarelamento ao longo do tempo, enquanto as polpas não-pasteurizadas apresentaram-se bem mais avermelhadas, porém sem muitas alterações ao longo do tempo.

Os valores do Cromaticidade (c) das polpas de acerola decresceram de 46,96, no início do armazenamento, para 37,91 ao final do armazenamento de 360 dias sob congelamento (Figura 2C).

Os valores do ângulo Hue (h), ou tonalidade de cor, das polpas de acerola não sofreram interação significativa entre tempo de armazenamento e tratamento (Figura 2D). O aumento do ângulo Hue mostra o amarelecimento das polpas dos frutos durante o armazenamento. Quanto maior este valor, mais amarelado torna-se o produto. Os valores do ângulo Hue nas polpas de acerola cresceram de 45,43, no início do armazenamento, para 64,82 , ao final do armazenamento de 360 dias sob congelamento. Este aumento pode ser atribuído provavelmente à degradação das antocianinas nas polpas. Observouse uma degradação de antocianinas elevada e crescente ao longo do armazenamento para ambas as polpas, pasteurizadas e não-pasteurizadas. Este fato também foi observado por ARAÚJO (2005), que evidenciou manutenção de cor nas polpas mais amareladas, entretanto, as polpas mais avermelhadas tornaram-se mais amareladas com o tempo de armazenamento.

As polpas pasteurizadas de acerola deste estudo apresentaram decréscimos nos valores médios de $\mathrm{pH}$ entre 3,22 e 3,27 (Figura 2E), resultados 

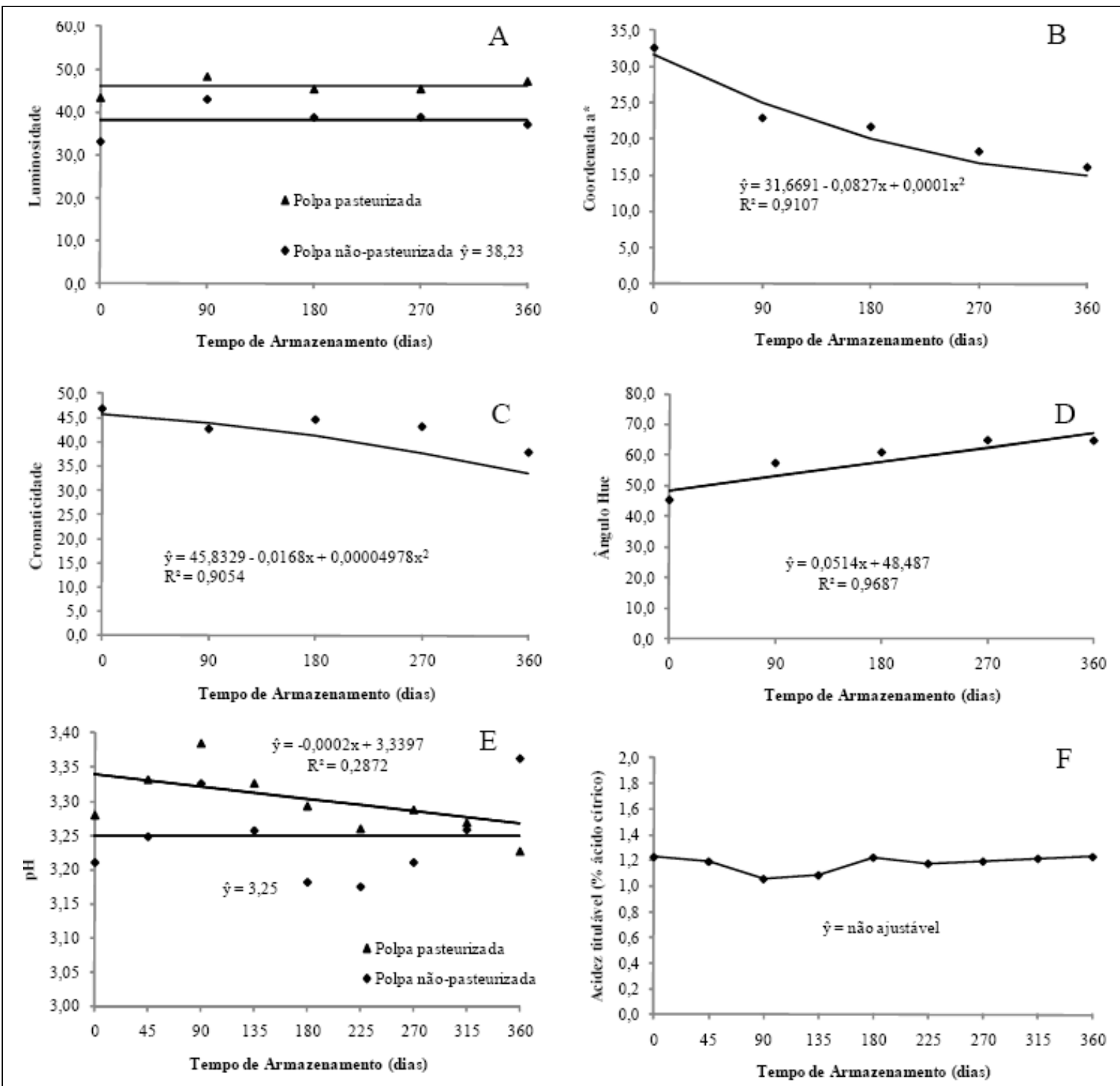

Figura 2 - Luminosidade (A), coordenada a* (B), cromaticidade (C), ângulo Hue (D), pH (E) e acidez titulável (F) de polpas de acerola oriundas de cultivo orgânico armazenadas a $-18^{\circ} \mathrm{C}$ por 360 dias.

semelhantes aos encontrados por SALGADO et al. (1999), com médias entre 3,12 a 3,44. OLIVEIRA (2008), estudando diferentes clones de aceroleira, observou que, nas polpas armazenadas a $-18^{\circ} \mathrm{C}$ por 11 meses, ocorreu uma pequena variabilidade nos valores de $\mathrm{pH}$, relatando médias entre 2,77 a 3,82. Resultados dessa autora corroboram os encontrados por ARAÚJO (2005), que observou pequena diminuição do $\mathrm{pH}$ em polpas de frutos de novos clones de aceroleira conservada por congelamento por 12 meses, ambos os frutos obtidos por sistema convencional de cultivo.

De acordo com SEMENSATO (1997), a acerola é uma fruta ácida com possibilidade de utilização industrial em geléias e doces, sem a necessidade de adição de ácidos no processamento. Portanto, a polpa de acerola aqui estudada é considerada como um alimento ácido $(\mathrm{pH}<4,5)$, garantindo assim sua segurança do ponto de vista microbiológico.

Os valores de acidez titulável variaram entre 1,24 a 1,15\% de ácido cítrico para polpas pasteurizadas e de 1,21 a 1,22\% de ácido cítrico para polpas nãopasteurizadas, apresentando-se estáveis ao longo do armazenamento (Figura 2F), evidenciando uma boa manutenção desse parâmetro. Mesmo as polpas que não sofreram tratamento térmico apresentaram-se com 
teores de ácido cítrico comparáveis aos produtos tratados com o calor. Possivelmente, a pasteurização não afetou a acidez do produto e o congelamento propiciou a estabilidade desse parâmetro.

Ocorreu um leve decréscimo no conteúdo de sólidos solúveis nas polpas estudadas até 360 dias de armazenamento, fato também observado por OLIVEIRA (2008) ao estudar armazenamento de polpas congeladas de diferentes clones de aceroleira a $-18^{\circ} \mathrm{C}$ por 11 meses, encontrando conteúdos que variaram de 6,10 a $9,17^{\circ}$ Brix. ARAÚJO (2007) também verificou a estabilidade no conteúdo de sólidos solúveis em polpas de frutos de clones de acerola conservada por congelamento por 12 meses.

Em estudos de caracterização de acerolas oriundas de cultivo convencional e orgânico, SILVA (2008) observou que os clones de aceroleira oriundos de cultivo orgânico apresentaram teores de sólidos solúveis superiores $\left(8,22\right.$ a $\left.11,02^{\circ} \mathrm{Brix}\right)$ aos de cultivo convencional $\left(5,83\right.$ a $\left.9,95^{\circ} \mathrm{Brix}\right)$, parecendo ter contribuído para sua maior concentração. Vale ressaltar que não existem muitos estudos na literatura relatando tal comportamento. Ao avaliar a composição química de sucos de acerola em diferentes estádios de maturação, RIGHETTO et al. (2005) observaram conteúdos superiores de sólidos solúveis em sucos de frutas maduras $\left(5,7^{\circ} \mathrm{Brix}\right)$ em relação à fruta verde $\left(5,1^{\circ} \mathrm{Brix}\right)$. As polpas pasteurizadas e não-pasteurizadas apresentaram, respectivamente, aumento de $2 \%$ e decréscimo de $1 \%$ no conteúdo de sólidos solúveis durante 360 dias de armazenamento.

Os teores de $\mathrm{pH}$, SS, AT e açúcares totais estão de acordo com o padrão da legislação brasileira para polpa de acerola (BRASIL, 2000), que estabelece limites aceitáveis de $\mathrm{pH}$ 2,80, 5,50 ${ }^{\circ}$ Brix, 0,80\% de acidez total e valor máximo de $9,5 \%$ para açúcares totais.

Um leve aumento da relação SS/AT foi observado para esta relação no final do armazenamento, fato devido à redução da acidez apresentada nas polpas. Segundo CHITARRA \& CHITARRA (2005), a relação SS/AT é uma das formas mais utilizadas para a avaliação do sabor, sendo mais representativa que a medição isolada de açúcares ou da acidez, indicando o grau de equilíbrio entre açúcares e ácidos orgânicos do fruto (MATSUURA et al., 2001). Esse parâmetro é importante do ponto de vista tecnológico, pois está diretamente relacionado à sua qualidade quanto ao atributo sabor, sendo mais atrativo para o consumo in natura.

SILVA(2008), estudando frutos de aceroleira provenientes de cultivo orgânico de quatro clones (71, AC 69, FP 19 e Okinawa) dos seis clones utilizados para obtenção da polpa utilizada neste estudo, encontrou relações de SS/AT similares às encontradas neste trabalho, sendo o AC 69 o que apresentou maior relação (6,69). ARAÚJO (2007) e OLIVEIRA(2008), ao avaliarem polpas de frutos de clones de aceroleira congeladas, obtidos de cultivo convencional, apresentaram mínimo e máximo de 3,73 e 7,33, e 4,67 e 7,09, respectivamente, com limite mínimo inferior e o máximo superior aos relatados neste experimento.

Os valores de açúcares totais tenderam a ficar constantes ao longo do armazenamento, com um leve aumento, de 4,07\% de glicose para $4,54 \%$ até os 360 dias. Esses compostos parecem ter contribuído para o alto teor de sólidos solúveis apresentados pelas polpas. CHITARRA \& CHITARRA(2005) afirmam que existe uma relação direta entre os sólidos solúveis e a concentração de açúcares solúveis totais. FREITAS et al. (2006) observaram pequenas variações no conteúdo de açúcar total em sucos de acerola obtidos pelo método hot fill, armazenadas à temperatura ambiente durante 350 dias de armazenamento.

\section{Análises microbiológicas}

A tabela 1 apresenta os resultados das análises microbiológicas realizadas nos produtos ao longo do período de armazenamento. Observa-se que, no início, ambas as polpas, pasteurizadas e nãopasteurizadas, apresentavam baixas contagens de coliformes totais e aeróbios mesófilos. O processo térmico utilizado para obtenção da polpa, a adequada característica sanitária dos frutos assim como a adequada condição higiênica de manuseio na indústria podem ter contribuído para tais resultados (MAIA et al., 2007). A acidez natural das polpas de acerola confere ao produto proteção contra microrganismos.

Segundo FRANCO \& LANDGRAF (2003), o emprego de tratamentos térmicos com temperaturas elevadas visando à conservação de alimentos fundamenta-se nos efeitos deletérios que o calor exerce sobre os microrganismos. A pasteurização torna-se um importante aliado na conservação de polpas por eliminar microrganismos patogênicos e também inativar enzimas. SOUSA et al. (2006) avaliaram sucos de açaí tratados termicamente e constataram resultados microbiológicos de acordo com a legislação. Todas as amostras apresentaram ausência de Salmonella sp. ao longo de todo o armazenamento (Tabela 1).

Em relação à contagem de bolores e leveduras, constatou-se a eficácia do processo para as polpas pasteurizadas, pois foram verificadas contagens inferiores desses microrganismos em relação às polpas não-pasteurizadas. As baixas contagens de microrganismos nas polpas possivelmente tenham sido em decorrência dos efeitos conjugados da aplicação de tratamento térmico (pasteurização), uso do 
Tabela 1 - Parâmetros microbiológicos de polpas de acerola oriundas de cultivo orgânico armazenadas a $-18^{\circ} \mathrm{C}$ por 360 dias.

\begin{tabular}{|c|c|c|c|c|c|c|c|c|}
\hline \multirow{2}{*}{$\begin{array}{l}\text { Tempo dearmazenamento } \\
\text { (dias) }\end{array}$} & \multicolumn{2}{|c|}{$\begin{array}{l}\text { Coliformes totais } \\
\qquad\left(\mathrm{NMP} \mathrm{g}^{-1}\right)\end{array}$} & \multicolumn{2}{|c|}{ Salmonella sp. } & \multicolumn{2}{|c|}{$\begin{array}{l}\text { Contagem de Aeróbios } \\
\text { mesófilos (UFC g }{ }^{1} \text { ) }\end{array}$} & \multicolumn{2}{|c|}{ 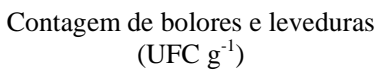 } \\
\hline & $\mathrm{PP}^{*}$ & $\mathrm{NP}^{* *}$ & PP & NP & PP & NP & PP & NP \\
\hline $\mathrm{T}_{0}$ & $<3$ & $<3$ & Aus. & Aus. & $3,8 \times 10$ & $4,6 \times 10^{2}$ & $<10$ & $1,3 \times 10^{2}$ \\
\hline $\mathrm{T}_{45}$ & $<3$ & $<3$ & Aus. & Aus. & $1,7 \times 10$ & $2,9 \times 10^{2}$ & $1,0 \times 10^{2}$ & $1,0 \times 10^{2}$ \\
\hline $\mathrm{T}_{90}$ & $<3$ & $<3$ & Aus. & Aus. & $<10$ & $6,2 \times 10^{2}$ & $<10$ & $1,0 \times 10^{2}$ \\
\hline $\mathrm{T}_{135}$ & $<3$ & $<3$ & Aus. & Aus. & $<10$ & $6,2 \times 10^{2}$ & $<10$ & $1,9 \times 10^{2}$ \\
\hline $\mathrm{T}_{180}$ & $<3$ & $<3$ & Aus. & Aus. & $2,2 \times 10^{2}$ & $5,1 \times 10^{2}$ & $<10$ & $<10$ \\
\hline $\mathrm{T}_{225}$ & $<3$ & $<3$ & Aus. & Aus. & $<10$ & $2,0 \times 10$ & $<10$ & $<10$ \\
\hline $\mathrm{T}_{270}$ & $<3$ & $<3$ & Aus. & Aus. & $<10$ & $<10$ & $<10$ & $<10$ \\
\hline $\mathrm{T}_{315}$ & $<3$ & $<3$ & Aus. & Aus. & $2,2 \times 10^{2}$ & $4,4 \times 10^{2}$ & $1,0 \times 10$ & $1,7 \times 10$ \\
\hline $\mathrm{T}_{360}$ & $<3$ & $<3$ & Aus. & Aus. & $1,8 \times 10^{2}$ & $3,2 \times 10^{2}$ & $1,0 \times 10$ & $1,0 \times 10$ \\
\hline
\end{tabular}

*PP - polpa pasteurizada; **PNP - polpa não-pasteurizada

congelamento e aplicação de envase asséptico que, juntos, podem apresentar efeito superior no controle da multiplicação de microrganismos quando utilizados isoladamente, conforme a teoria dos obstáculos, proposta por LEISTNER (1994).

\section{CONCLUSÃO}

O tratamento térmico contribuiu negativamente nas características sólidos solúveis e açúcares solúveis totais e redutores, reduzindo seus conteúdos iniciais. As polpas pasteurizadas e nãopasteurizadas apresentaram boa qualidade microbiológica do início ao final do armazenamento, com todos os resultados obtidos de acordo com as determinações estabelecidas pela Resolução RDC nํㅜ 12, de 02 de janeiro de 2001 e Instrução Normativa no 1 1, de 07 de janeiro de 2000. Dentre as polpas estudadas, as polpas não-pasteurizadas apresentaram melhores características iniciais de cor. Já as polpas pasteurizadas garantiram melhores características microbiológicas, no que concerne aos aspectos de segurança alimentar.

\section{REFERÊNCIAS}

AOAC - ASSOCIATION OF OFFICIAL ANALYTICAL CHEMISTRY. Official methods of Analysis of the Association of Official Analytical Chemistry. 15.ed. Washington, 1995. 2V.

APHA - AMERICAN PUBLIC HEALTH ASSOCIATION. Compendium of methods for the microbiological examination of foods. Washington, DC, 2001. 676p.

ARAÚJO, P.G.L. Conservação pós-colheita e estabilidade da polpa congelada de acerolas Apodi, Cereja, Frutacor, II 47/1, Roxinha e Sertaneja. 2005. 67f. Dissertação
(Mestrado em Tecnologia de Alimentos) - Universidade Federal do Ceará, Fortaleza, CE.

ARAÚJO, P.G.L. et al. ß-Caroteno, ácido ascórbico e antocianinas totais em polpa de frutos de aceroleira conservada por congelamento durante 12 meses. Ciênc Tecnol Aliment, Campinas, v.27, n.l, p.104-107, 2007. Disponível em: <http:/ /www.scielo.br/scielo.php?script=sci_arttext\&pid=S010120612007000100018>. Acesso em: 25 set. 2010. doi: 10.1590/ S0101-20612007000100018.

BORGES, A.L. et al. Cultivo orgânico de fruteiras tropicais - manejo do solo e da cultura. Cruz das Almas: EMBRAPACNPMF, 2003. (Comunicado Técnico 64).

BRASIL. Ministério da Saúde. Agência Nacional de Vigilância Sanitária. Métodos físicos-químicos para análise de alimentos. Brasília: Ministério da Saúde, 2005. 1018p.

BRASIL. Ministério da Agricultura e do Abastecimento. Instrução Normativa n.01, de 07 de janeiro de 2000. Regulamento técnico geral para fixação dos padrões de qualidade para polpa de fruta. Diário Oficial da União n.6. Brasília, 10 de janeiro de 2000. Seção I. p.54-58.

BRUNINI, M.A. et al. Caracterização física e química de acerolas provenientes de diferentes regiões de cultivo. Rev Bras de Frutic, Jaboticabal, v.26, n.3, p.486-489, 2004. Disponível em: <http://www.scielo.br/ scielo.ph p ? script = sci_art text \& pid = S 0100 29452004000300027>. Acesso em: 20 set. 2010. doi: 10.1590/ S0100-29452004000300027.

ChITARRA, M.I.F.; CHITARRA, A.B. Pós-colheita de frutas e hortaliças: fisiologia e manuseio. Lavras: UFLA, 2005. 785p.

DINIZ, E. et al. Atividade de água e condutividade elétrica de polpas de acerola concentradas. Rev Bras Prod Agroindustr, Campina Grande, n.1, p.9-17, 2003. Disponível em: <http:// www.deag.ufcg.edu.br/rbpa/rev5e/Art5e2.pdf $>$. Acesso em: 24 set. 2010. 
FREITAS, C.A.S. et al. Storage stability of acerola tropical fruit juice obtained by hot fill method. Intern J Food Sci Tech, v. 41, p.1216-1221, 2006. Disponível em: <http:// dx.doi.org/10.1111/j.1365-2621.2006.01188.x>. Acesso em: 17 ago. 2010. doi: 10.1111/j.1365-2621.2006.01188.x.

FRANCO, B.D.G.M.; LANDGRAF, M.L.S. Microbiologia dos alimentos. São Paulo: Atheneu, 2003. 182p.

LEISTNER, L. Food design by hurdle techonology and HACCP. Kulmbach: Adalbet Raps Foundation, 1994. 62p.

MAIA, G.A. et al. Processamento de sucos de frutas tropicais. Fortaleza: UFC, 2007. 320p.

MATSUURA, F.C.A.U. et al. Avaliações físico-químicas em frutos de diferentes genótipos de acerola (Malpighia punicifólia D.C.). Rev Bras Frutic, Jaboticabal, v.23, n.3, p.602-606, 2001. Disponível em: <http://www.scielo.br/ scielo.php? script =sci_art text \& pid=S 0100 $29452001000300032 \& \operatorname{lng}=p t \& n r m=i s o \& t \operatorname{lng}=p t>$. Acesso em: 24 set. 2010. doi: 10.1590/S0100-29452001000300032.

MILLER, G.L. Use of dinitrosalicylic acid reagent for determination of reducing sugar. Anal Biochem New York, v.31, p.426-428, 1959. Disponível em: <http://dx.doi.org/ 10.1021/ac60147a030>. Acesso em: 25 set. 2010. doi: $10.1021 / \mathrm{ac} 60147 \mathrm{a} 030$

OLIVEIRA, L.S. Avaliação da qualidade pós-colheita e capacidade antioxidante durante $o$ armazenamento da polpa de seis clones de aceroleira. 2008. 93f. Dissertação (Mestrado em Bioquímica) - Universidade Federal do Ceará, Fortaleza, CE.

RIGHETTO, A.M. et al. Chemical composition and antioxidant activity of juices from mature and immature acerola (Malpighia emarginata DC). Food Sci Technol, London, v.11, n.4, p.315321, 2005. Disponível em: <http://dx.doi.org/10.1177/
1082013205056785>. Acesso em: 24 set. 2010. doi: 10.1177/ 1082013205056785 .

SALGADO, S.M. et al. Polpa de fruta congelada: efeito do processamento sobre o conteúdo de fibra alimentar. Rev Nutr, Campinas, v.12, n.3, p.303-308, 1999. Disponível em: <http:/ /www.scielo.br/scielo.php?script=sci_arttext\&pid=S1415$52731999000300009 \& \operatorname{lng}=p t \& n r m=i s o \&$ tlng $=p t>$. Acesso em: 24 set. 2010. doi: 10.1590/S1415-52731999000300009.

SAS Institute, Inc. SAS user's guide: version 9.1. Cary, NC, 2006. 180p.

SEMENSATO, L.R. Caracterização físico-química de frutos de genótipos de acerola (Malpighia sp.), cultivados em Anápolis-GO, processamento e estabilidade de seus produtos. 1997. 74f. Dissertação (Mestrado em Fitotecnia) - Universidade Federal de Goiás, Goiânia, Go.

SILVA, W.S. Qualidade e atividade antioxidante em frutos de variedades de aceroleira. 2008. 134f. Dissertação (Mestrado em Tecnologia de Alimentos) - Centro de Ciências Agrárias, Universidade Federal do Ceará, Fortaleza, CE.

SOARES, E.C. et al. Desidratação da polpa de acerola (Malpighia emarginata D. C.). Ciênc Tecnol Aliment, Campinas, v.21 n. 2 p.164-170, 2001. Disponível em: <http:/ / w w w.scielo.br/scielo.ph p ? pid= S 0101 20612001000200008\&script=sci_arttext $>$. Acesso em: 25 set. 2010. doi: 10.1590/S0101-20612001000200008.

SOUSA, M.A.C. et al. Suco de açaí (Euterpe oleracea Mart.): avaliação microbiológica, tratamento térmico e vida de prateleira. Acta Amazonica, v.36, n.4, p.483-496, 2006. Disponível em: <http://www.scielo.br/ scielo.php? script =sci_arttext \& pid= S004459672006000400010>. Acesso em: 25 set. 2010. doi: 10.1590/ S0044-59672006000400010>. 